\title{
Performance Evaluation of Integrated Wireless Networks with Virtual Partition of Channels
}

\author{
Agassi Melikov ${ }^{1}$, Mehriban Fattakhova $^{2}$, Gulnara Velidzanova $^{2}$, \\ and János Sztrik ${ }^{3}$ \\ 1 National Aviation Academy, Azerbaijan \\ agassi.melikov@rambler.ru \\ 2 Institute of Cybernetics, National Academy of Sciences, Azerbaijan \\ meri-fattax@mail.ru, gulnaravelicanova@rambler.ru \\ 3 University of Debrecen, Hungary \\ sztrik.janos@inf .unideb.hu
}

\begin{abstract}
A new access scheme in integrated wireless networks which is based on virtual partition of channels among voice and data calls is proposed. In this scheme a voice call occupies a free channel in its own zone and if there is no available channel in the given zone a handover voice call searches for an idle channel in another zone. A threshold for number of handover voice calls in zone of channels for data calls is defined. To determine the access scheme of new data calls a state-dependent threshold based rule is introduced. An effective method to calculate the QoS metrics of the defined access scheme is developed. Some sample results illustrating the numerical experiments are collected and analyzed.
\end{abstract}

Keywords: integrated networks, voice and data calls, partition of channels, quality of service metrics, calculation method.

\section{Introduction}

In the last few decades the teletraffic theory has become a very important and effective scientific discipline representing a set of probabilistic methods to solve problems of designing and optimization of telecommunication systems. Information technology solutions require analytical, numerical, approximate, simulation and hybrid techniques. The current state of mathematical theory of the teletraffic problems has been collected in a detailed review [1. That paper and other ones justify that performance evaluation of wireless networks plays a central role, see for example [2], 4], 5], 8], 13. Developing effective methods to calculate QoS metrics of integrated cellular networks under various Call Admission Control (CAC) schemes are important, see [10], [12].

Main goals of any $\mathrm{CAC}$ when determining the rules is to use the scarce resources (frequencies, time slots, codes and their combinations). These rules are necessary to prevent (or minimization) the conflict situations due to employment of specified resources as well as to satisfy the desired QoS level for heterogeneous calls.

A. Dudin et al. (Eds.): ITMM 2014, CCIS 487, pp. 269-276 2014.

(C) Springer International Publishing Switzerland 2014 
In an integrated cellular network calls of real-times (e.g. voice calls) and nonreal times (e.g. data calls) are distinguished. In such networks either CAC based on guard channels scheme or CAC based on cut-off scheme are used. In both schemes all channels are available for calls of any type.

To reduce the possibility of conflict situations the schemes which are based on the partition of pool of channels between heterogeneous calls are more useful. Literature review shows that models of integrated cellular networks with such kind of access schemes are insufficiently investigated.

Note that fixed (rigid or isolated) partition of channels is not effective one as noted by [6] and thus other schemes are required. It should be underlined that non-isolated schemes of partition of channels in networks with single traffic (a network of the second generation) have been offered in [9] and in Chapter 1 of the book 11] (pp. 18 and 19). The main contribution is that in these schemes the partition of channels is not rigid, i.e. the scheme of virtual partition of channels (Virtual Partitioning, VP) is suggested.

In the present paper a multi-parametric VP-scheme for partition of channels in integrated cellular networks is proposed. Exact formulas to calculate QoS metrics of such CAC scheme are developed. Figures generated by results of several sample numerical experiments are shown and analyzed.

\section{VP-scheme of Partition}

A base station of integrated cellular network contains of $N>1$ radio channels. These channels are divided into two parts: a number of $N_{v}$ channels is assigned for voice calls only and the remaining $N_{v d}=N-N_{v}$ channels are used by both voice and data calls. In other words, the pool of channels is divided into individual zone with $N_{v}$ channels (for voice calls only) and common one with $N_{v d}$ channels (both for voice and data calls).

Virtual partition means the following: on termination of processing of the $\mathrm{v}$-call in a $v$-zone, the released channel is transferred into the $v d$-zone if there is a $v$-call in that zone and simultaneously the channel in a $v d$-zone which process a $v$-call, is transferred into the $v$-zone.

In this network four types of Poisson-type arrival traffics, i.e. new (ov-calls) and handover voice calls ( $h v$-calls), furthermore new (od-calls) and handover data calls ( $h d$-calls) are assumed. Intensity of $x$-calls is $\lambda_{x}, x \in\{o v, h v, o d, h d\}$, respectively.

Distribution function of channel holding time for both kind of calls is supposed to be exponential the mean for voice calls (new or handover) is $1 / \mu_{v}$, and the corresponding parameter for data calls (new or handover) is $1 / \mu_{d}$. Identity of channel holding times for new and handover calls of both types is explained by the memoryless property of the exponential distribution.

Access of $v$-calls is specified by the following rules:

- If upon arrival an ov-call, there is free channel in $v$-zone, then it is accepted; otherwise, it is rejected. 
- If upon arrival an $h v$-call, there is free channel in $v$-zone, then it is accepted; otherwise, free channel is searched in $v d$-zone. At that limit to maximum number of $h v$-calls in $v d$-zone is defined, i.e. maximum number of $h v$-calls in $v d$-zone is $R_{h v}, 1 \leq R_{h v} \leq N_{v d}$. If at the moment of arriving an $h v$-call, number of $h v$-calls in $v d$-zone is equal $R_{h v}$, then it is rejected. Note that the average channel holding time for $h v$-calls in $v d$-zone is $1 / \mu_{v}$, too.

Access of $d$-calls is specified by the following rules:

- If upon arrival an $h d$-call, there is free channel in $v d$-zone, then it is accepted; otherwise, it is rejected.

- If upon arrival an od-call, the number of $d$-calls in $v d$-zone is less than $R_{o d}, 1 \leq R_{o d} \leq N_{v d}-1$, then it is accepted; otherwise, it is rejected.

The primary goal of our investigation is to find the main QoS metrics of this system, namely, the loss probabilities of calls for each type.

\section{Method to Solve the Problem}

The state of a cell is described by a two-dimensional vector $\boldsymbol{n}=\left(n_{d}, n_{v}\right)$ where $n_{d}$ and $n_{v}$ denote the total number of data calls and voice calls, respectively. Then the state space of the corresponding two-dimensional Markov chain (2-D MC) is defined as follows:

$$
S=\left\{\boldsymbol{n}: n_{d}=0,1, \ldots, N_{v d} ; \quad n_{v}=0,1, \ldots, N_{v}+R_{h v} ; \quad n_{d}+n_{v} \leq N\right\} .
$$

According to the introduced access scheme, non-negative elements of generating matrix ( $Q$-matrix) are determined from the following relationships:

$$
q\left(\boldsymbol{n}, \boldsymbol{n}^{\prime}\right)= \begin{cases}\lambda_{d} & \text { if } n_{d}<R_{o d}, \quad \boldsymbol{n}^{\prime}=\boldsymbol{n}+\boldsymbol{e}_{1}, \\ \lambda_{h d} & \text { if } n_{d} \geq R_{o d}, \quad \boldsymbol{n}^{\prime}=\boldsymbol{n}+\boldsymbol{e}_{1}, \\ \lambda_{v} & \text { if } n_{v}<N_{v}, \boldsymbol{n}^{\prime}=\boldsymbol{n}+\boldsymbol{e}_{2}, \\ \lambda_{h v} & \text { if } N_{v} \leq n_{v}<N_{v}+R_{h v}, \quad \boldsymbol{n}^{\prime}=\boldsymbol{n}+\boldsymbol{e}_{2}, \\ n_{d} \mu_{d} & \text { if } \boldsymbol{n}^{\prime}=\boldsymbol{n}-\boldsymbol{e}_{1}, \\ n_{v} \mu_{v} & \text { if } \boldsymbol{n}^{\prime}=\boldsymbol{n}-\boldsymbol{e}_{2}, \\ 0 & \text { in other cases, }\end{cases}
$$

where $\lambda_{v}=\lambda_{o v}+\lambda_{h v}, \quad \lambda_{d}=\lambda_{o d}+\lambda_{h d}, \quad e_{1}=(1,0), \quad e_{2}=(0,1)$.

It is easy to show that given finite 2 -D is irreducible, so in this chain equilibrium regime exists. Let $p(\boldsymbol{n})$ denote the stationary probability of state $\boldsymbol{n} \in S$.

The above-mentioned QoS metrics are determined as appropriate marginal distributions of the defined 2-D MC.

Let $P_{x}$ be the loss probability of $x$-calls, $x \in\{h v, o v, h d, o d\}$. Taking into account described above rules for accepting of heterogeneous calls and by using PASTA-theorem [14] we obtain the following formulas to calculate the QoS metrics of the network: 


$$
\begin{aligned}
P_{o v}= & \sum_{\boldsymbol{n} \in S} p(\boldsymbol{n}) I\left(n_{v} \geq N_{v}\right) \\
P_{o d}= & \sum_{\boldsymbol{n} \in S} p(\boldsymbol{n}) I\left(n_{d} \geq R_{o d}\right) \\
P_{h d}= & \sum_{\boldsymbol{n} \in S} p(\boldsymbol{n}) \delta\left(n_{d}+n_{v}, N_{v d}\right) ; \\
P_{h v}= & \sum_{\boldsymbol{n} \in S} p(\boldsymbol{n})\left(\delta\left(n_{v}, R_{h v}\right)\left(1-\delta\left(n_{d}+n_{v}, N_{v d}\right)\right)+\right. \\
& +\left(1-\delta\left(n_{v}, R_{h v}\right) \delta\left(n_{d}+n_{v}, N_{v d}\right)\right) ;
\end{aligned}
$$

where $\delta(i, j)$ are Kronecker's symbols, $I(A)$ is the indicator function of event $A$. Thus, to calculate these QoS metrics, as usual the solution of system of global balance equations (SGBE) is required. In general, due to the large state space we face to the state space explosion problem. However, in this case the considered SGBE has analytical solution in multiplicative form. Namely, we have

Proposition. The stationary distribution has the following multiplicative form:

Case $R_{o d} \leq N_{v d}-R_{h v}$ :

$$
\begin{aligned}
& p(i, j) \\
& = \begin{cases}\frac{v_{d}^{i}}{i !} \frac{v_{v}^{j}}{j !} p(0,0) & \text { if } 0 \leq i \leq R_{o d}, 0 \leq j \leq N_{v}, \\
\left(\frac{v_{d}}{v_{h d}}\right)^{R_{o d}} \frac{v_{h d}^{i}}{i !} \frac{v_{v}^{j}}{j !} p(0,0) & \text { if } R_{o d}+1 \leq i \leq N_{v d}, 0 \leq j \leq N_{v} \\
\left(\frac{v_{v}}{v_{h v}}\right)^{N_{v}} \frac{v_{d}^{i}}{i !} \frac{v_{h v}^{j}}{j !} p(0,0) & \text { if } 0 \leq i \leq R_{o d}, N_{v}+1 \leq j \leq N_{v}+R_{h v} \\
\left(\frac{v_{d}}{v_{h d}}\right)^{R_{o d}}\left(\frac{v_{v}}{v_{h v}}\right)^{N_{v}} \frac{v_{h d}^{i}}{i !} \frac{v_{h v}^{j}}{j !} p(0,0) & \text { if } R_{o d}+1 \leq i \leq N_{v d}-1, \\
& N_{v}+1 \leq j \leq \min \left(N_{v}+R_{h v}, N-i\right)\end{cases}
\end{aligned}
$$

Case $R_{o d}>N_{v d}-R_{h v}$ :

$$
\begin{aligned}
& p(i, j) \\
& =\left\{\begin{array}{lc}
\frac{v_{d}^{i}}{i !} \frac{v_{v}^{j}}{j !} p(0,0) & \text { if } 0 \leq i \leq R_{o d}, 0 \leq j \leq N_{v}, \\
\left(\frac{v_{d}}{v_{h d}}\right)^{R_{\text {od }}} \frac{v_{h d}^{i}}{i !} \frac{v_{v}^{j}}{j !} p(0,0) & \text { if } R_{o d}+1 \leq i \leq N_{v d}, 0 \leq j \leq N_{v}, \\
\left(\frac{v_{v}}{v_{h v}}\right)^{N_{v}} \frac{v_{d}^{i}}{i !} \frac{v_{h v}^{j}}{j !} p(0,0) & \text { if } 0 \leq i \leq R_{o d}, N_{v}+1 \leq j \leq \\
\left(\frac{v_{d}}{v_{h d}}\right)^{R_{o d}}\left(\frac{v_{v}}{v_{h v}}\right)^{N_{v}} \frac{v_{h d}^{i}}{i !} \frac{v_{h v}^{j}}{j !} p(0,0) & \text { if } \begin{array}{c}
\left.R_{o d}+1 \leq i \leq N_{v}+R_{h v}, N-i\right), \\
N_{v}+1 \leq j \leq N-i ;
\end{array}
\end{array}\right.
\end{aligned}
$$

In both formulas as usual $p(0,0)$ is determined by the normalizing condition. 
Proof of this fact is based on Kolmogorov's theorem about reversibility of 2-D MC, see for example [7. Indeed, it is easily shown that there is no circulation between states $\boldsymbol{n}, \boldsymbol{n}+\boldsymbol{e}_{1}, \boldsymbol{n}+\boldsymbol{e}_{2}, \boldsymbol{n}+\boldsymbol{e}_{1}+\boldsymbol{e}_{2}$ of the state diagram of the underlying 2-D MC. Indeed, in both cases $R_{o d} \leq N_{v d}-R_{h v}$ and $R_{o d}>N_{v d}-R_{h v}$ circulation flow among the indicated four states in both directions (clockwise and counter clockwise) is equals $\lambda_{d} \lambda_{v}\left(n_{d}+1\right) \mu_{d}\left(n_{v}+1\right) \mu_{v}$. In other words, system of local balance equations (SLBE) is fulfilled, i.e. there is a general solution of the SLBE for state probabilities. Thus by choosing the path $(0,0),(1,0),,(i, 0),(i, 1),,(i, j)$ from state $(0,0)$ to state $(i, j)$ we find that multiplicative solution (7) (or (8)) is hold. Note that in this proof scheme it is required take into account four cases in formulas (7) and (8) which are indicated in the right sides of the indicated formulas.

Now we are ready to obtain the respective loss probabilities in the following explicit formulas

$$
\begin{aligned}
P_{o v} & =\sum_{i=0}^{N_{v d}-R_{h v}} \sum_{j=N_{v}}^{N_{v}-R_{h v}} p(i, j)+\sum_{i=N_{v d}-R_{h v}+1}^{N_{v d}} \sum_{j=N_{v}}^{N-i} p(i, j) ; \\
P_{h v} & =\sum_{i=0}^{N_{v d}-R_{h v}} p\left(i, N_{v}+R_{h v}\right)+\sum_{i=N_{v d}-R_{h v}+1}^{N_{v d}} p(i, N-i) ; \\
P_{o d} & =\sum_{i=R_{o d}}^{N_{v d}} \sum_{j=0}^{\min \left(N_{v}+R_{h v} N-i\right)} p(i, j) ; \\
P_{h d} & =\sum_{i=0}^{N_{v}-1} p\left(N_{v d}, i\right)+\sum_{i=N_{v d}-R_{h v}}^{N_{v d}} p(i, N-i) .
\end{aligned}
$$

\section{Numerical Results}

The developed above explicit formulas allow us to investigate behavior of QoS metrics of the proposed partition scheme over any range of change of values of loading parameters of heterogeneous calls and number of channels. First of all, here it is assumed that allocation of entire pool of channels between zones is fixed and only regulated parameters are $R_{h v}$ and $R_{o d}$. It is clear that the increase in value of one of the parameters $R_{h v}$ and $R_{o d}$ (in an admissible area) favorably influences the QoS metric of calls of the corresponding type only.

The initial data for total number of channels and loading parameters of heterogeneous calls are as in 3 , i.e.

$$
N=30, \quad \lambda_{o}+\lambda_{h}=0.15, \quad \lambda_{o d}+\lambda_{h d}=0.3, \quad \mu_{v}^{-1}=2, \quad \mu_{d}^{-1}=120 .
$$

Below assume that $N_{v}=12, \quad N_{v d}=18$ and $30 \%$ of the total intensity of voice calls are handover voice calls and $80 \%$ of the total intensity of data calls are new data calls. 


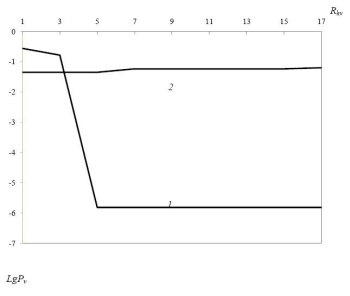

Fig. 1. $P_{v}$ vs $R_{h v} ; 1-P_{h v}, \quad 2-P_{o v}$

Consider the results of numerical experiments for the model with VP-scheme for partition of channels. In Fig. 1 the dependency of QoS metrics on the parameter $R_{h v}$ is shown. It is seen from Fig. 1 that function $P_{h v}$ decreases in small values of parameter $R_{h v}$ with high speed, thereafter it becomes almost constant; function $P_{o v}$ increases with insignificant speed in small values of indicated parameter, thereafter it becomes almost constant also. Almost constants are both functions $P_{o d}$ and $P_{h d}$ versus $R_{h v}$ (see Fig. 2). Such behavior of functions $P_{o d}$ and $P_{h d}$ is explained via small intensity of handover voice calls.

Dependency of QoS metrics on the parameter $R_{o d}$ are shown in Figs. 3 and 4. Here both functions $P_{o v}$ and $P_{h v}$ increases with insignificant speed in small values of indicated parameter, thereafter it becomes almost constant (see Fig. 3). However, function $P_{o d}$ decreases with significant speed versus $R_{o d}$ while function $P_{h d}$ is almost constant one (see Fig. 41).

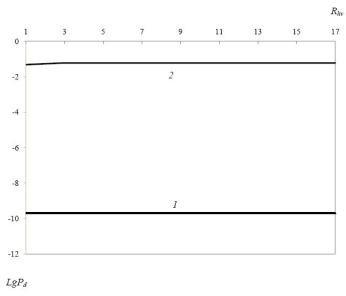

Fig. 2. $P_{d}$ vs $R_{h v} ; \quad 1-P_{h d}, \quad 2-P_{o d}$

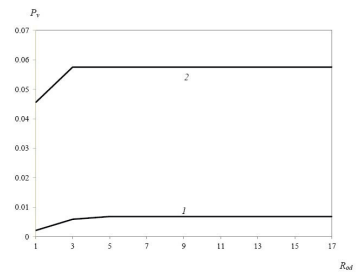

Fig. 3. $P_{v}$ vs $R_{o d} ; \quad 1-P_{h v}, \quad 2-P_{o v}$ 
It should be noted that as these numerical results show that all QoS metrics have monotony property. These facts allow us to develop the algorithms to find the set of effective values in order to satisfy the given QoS level.

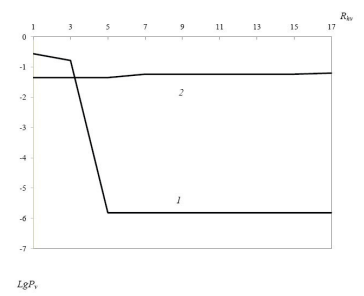

Fig. 4. $P_{d}$ vs $R_{o d} ; \quad 1-P_{o d}, \quad 2-P_{h d}$

\section{Conclusions}

In this paper, a virtual scheme to partition of entire pool of channels of isolated cell in integrated wireless networks was proposed. In accordance to this scheme all channels are virtually distributed between voice and data calls and there are limits to the number of handover voice calls and new data calls in zone of channels for data calls. The indicated limits are state-dependent parameters.

Explicit formulas to calculate loss probabilities of the network under given partition scheme were developed. The obtained formulas allow us to solve the problems related to satisfy desired QoS level of heterogeneous calls. These problems are subject of future research.

Acknowledgments. The work of A. Melikov, M.Fattakhova and G. Velidzanova was supported by Science Development Foundation under the President of the Republic of Azerbaijan - Grant No. EIF-RITN-MQM-2/IKT-2-2013$7(13)-29 / 01 / 1$.

The work of János Sztrik was realized in the frames of TÁMOP 4.2.4. A/2-111-2012-0001 National Excellence Program - Elaborating and operating an inland student and researcher personal support system. The project was subsidized by the European Union and co-financed by the European Social Fund.

\section{References}

1. Basharin, G.P., Samouylov, K.E., Yarkina, N.V., Gudkova, I.A.: A new stage in mathematical teletraffic theory. Automation Remote Control 70(12), 1954-1964 (2009)

2. Bérczes, T., Almási, B., Kuki, A., Sztrik, J.: The effect of RF unit breakdowns in sensor communication networks. Infocommunications Journal 5, 11-16 (2013)

3. Carvalho, G.H.S., Martins, V.S., Frances, C.R.L., Costa, J.C.W.A., Carvalho, S.V.: Performance analysis of multi-service wireless network: An approach integrating cac, scheduling, and buffer management. Comput. Electric. Eng. 34, 346-356 (2008) 
4. Daigle, J.N.: Queuing theory with applications to packet telecommunication. Springer, New York (2005)

5. Van Do, T., Chakka, R., Sztrik, J.: Spectral expansion solution methodology for QBD-M processes and applications in Future Internet engineering. In: Nguyen, N.T., van Do, T., Thi, H.A. (eds.) ICCSAMA 2013. SCI, vol. 479, pp. 131-142. Springer, Heidelberg (2013)

6. Feng, W., Kowada, M.: Performance analysis of wireless mobile networks with queueing priority and guard channels. International Transactions on Operational Research 15, 481-508 (2008)

7. Kelly, F.P.: Reversibility and stochastic networks. John Wiley \& Sons, New York (1979)

8. Lakatos, L., Szeidl, L., Telek, M.: Introduction to queuing systems with telecommunication applications. Springer, Heidelberg (2013)

9. Melikov, A.Z., Fattakhova, M.I., Babayev, A.T.: Investigation of cellular communication networks with private channels for service of handover calls. Automatic Control and Computer Sciences 39(3), 61-69 (2005)

10. Oh, Y., Kim, C.S., Melikov, A.Z., Fattakhova, M.I.: Numerical analysis of multiparameter strategy of access in multiservice cellular communication networks. Automation and Remote Control 71(12), 2558-2572 (2010)

11. Ponomarenko, L., Kim, C.S., Melikov, A.: Performance analysis and optimization of multi-traffic on communication networks. Springer, Heidelberg (2010)

12. Schneps-Schneppe, M., Iversen, V.B.: Call admission control in cellular networks. In: Ortiz, J.H. (ed.) Mobile Networks, pp. 111-136. Intech (2012)

13. Stasiak, M., Glabowski, M., Wishniewski, A., Zwierzykowski, P.: Modeling and dimensioning of mobile networks. John Wiley, Chichester (2011)

14. Wolff, R.W.: Poisson arrivals see time averages. Operations Research 30(2), 223$231(1992)$ 